\title{
Relationship between molecular response and quality of life with bosutinib or imatinib for chronic myeloid leukemia
}

\author{
Tim H. Brümmendorf ${ }^{1}$ • Carlo Gambacorti-Passerini ${ }^{2} \cdot$ Andrew G. Bushmakin $^{3}$ • Joseph C. Cappelleri ${ }^{3}$. \\ Andrea Viqueira $^{4} \cdot$ Arlene Reisman $^{5} \cdot$ Susanne Isfort ${ }^{1} \cdot$ Carla Mamolo $^{3}$
}

Received: 31 January 2020 / Accepted: 30 March 2020 / Published online: 19 April 2020

(C) The Author(s) 2020

\begin{abstract}
Patients with newly diagnosed chronic phase chronic myeloid leukemia (CP CML) can be effectively treated with tyrosine kinase inhibitors (TKIs) and achieve a lifespan similar to the general population. The success of TKIs, however, requires long-term and sometimes lifelong treatment; thus, patient-assessed health-related quality of life (HRQoL) has become an increasingly important parameter for treatment selection. Bosutinib is a TKI approved for CP CML in newly diagnosed adults and in those resistant or intolerant to prior therapy. In the Bosutinib Trial in First-Line Chronic Myelogenous Leukemia Treatment (BFORE), bosutinib demonstrated a significantly higher major molecular response rate compared with imatinib, with maintenance of HRQoL (measured by the Functional Assessment of Cancer Therapy-Leukemia (FACT-Leu) questionnaire), after 12 months of firstline treatment. We examined relationships between molecular response (MR) and HRQoL. MR values were represented by a logreduction scale (MRLR; a continuous variable). A repeated-measures longitudinal model was used to estimate the relationships between MRLR as a predictor and each FACT-Leu domain as an outcome. Effect sizes were calculated to determine strength of effects and allow comparisons across domains. The majority of FACT-Leu domains (with the exception of social well-being and physical well-being) demonstrated a significant relationship with MRLR $(p<0.05)$. Our results showed variable impact of clinical improvement on different dimensions of HRQoL. For patients who achieved $\mathrm{MR}^{5}$, emotional well-being and leukemia-specific domains showed the greatest improvement, with medium differences in effect sizes, whereas social wellbeing and physical well-being had the weakest relationship with MR.
\end{abstract}

Keywords Chronic myeloid leukemia $\cdot$ Health-related quality of life $\cdot$ Molecular response $\cdot$ Bosutinib $\cdot$ Imatinib

\section{Introduction}

Tyrosine kinase inhibitors (TKIs) that block the activity of the $B C R-A B L 1$ gene fusion product, the hallmark of chronic myeloid leukemia (CML), have substantially improved efficacy and tolerability of treatment and extended patient life expectancy to nearly that of the general population [1]. In clinical

Tim H. Brümmendorf

tbruemmendorf@ukaachen.de

1 Universitätsklinikum RWTH Aachen, Pauwelsstrasse 30, 52074 Aachen, Germany

2 University of Milano-Bicocca, Monza, Italy

3 Pfizer Inc, Groton, CT, USA

4 Pfizer SLU, Madrid, Spain

5 Pfizer Inc, New York, NY, USA trials that compared second-generation TKIs (bosutinib, dasatinib, or nilotinib) with the first-generation TKI imatinib in newly diagnosed patients with chronic phase (CP) CML, the second-generation TKIs showed superior efficacy, defined as cytogenetic or molecular responses (MR) at or by 12 months of treatment, versus imatinib [2-4]. Despite achievement of treatment-free remission becoming an increasingly well-established goal of first-line treatment $[5,6]$, the majority of patients with CP CML still require lifelong TKI therapy; thus, preserving or improving health-related quality of life (HRQoL) has become an important consideration for treatment selection.

To date, prospective assessment of patient-reported HRQoL in CML trials of most first- and second-generation TKIs has been scarce [7]. However, patient-reported outcome (PRO) data from clinical studies of bosutinib have indicated that patients with CML experienced stable or, in some cases, improved HRQoL during treatment compared with 
pretreatment status [8-11]. Relationships between distinct side effects of individual TKIs and HRQoL have been explored, e.g., PROs in patients with CML who experienced diarrhea during treatment with bosutinib [10, 12], but associations between efficacy and HRQoL are largely unknown.

Approval of bosutinib for newly diagnosed patients with CP CML was based on data from the ongoing, randomized, phase 3 BFORE trial, which demonstrated a significantly higher major MR (MMR) rate at 12 months in the modified intent-to-treat (ITT) population (primary endpoint) with bosutinib $(n=246)$ versus imatinib $(n=241)$ [2]. After longer follow-up ( $\geq 24$ months), bosutinib continued to demonstrate improved efficacy compared with imatinib, as evidenced by a higher cumulative MMR rate $(68.7 \%$ vs. $59.3 \%$; odds ratio, $1.51 ; 95 \%$ confidence interval, $1.06-2.16)$ and $\mathrm{MR}^{4}$ rate (39.9\% vs. $31.3 \%$; odds ratio, $1.45 ; 95 \%$ confidence interval, $1.02-2.07$ ) in the ITT population (bosutinib, $n=268$ and imatinib, $n=268$ ) at any time on treatment (Pfizer Inc, data on file) [13]. Treatment-emergent adverse events after $\geq$ 24 months' follow-up were consistent with the known safety profiles of bosutinib [14] and imatinib [15]; diarrhea and transaminase increases were more frequent with bosutinib, and musculoskeletal events were more common with imatinib (Pfizer Inc., data on file). Assessment of PROs with the Functional Assessment of Cancer Therapy-Leukemia (FACT-Leu) questionnaire was an exploratory objective of the BFORE trial; after 12 months of treatment with either bosutinib or imatinib, patients in the modified ITT population maintained or improved HRQoL compared with baseline [11]. Repeated-measures mixed-effects modeling showed no significant differences in HRQoL at month 12 with bosutinib versus imatinib [11].

Here, we examined the relationships between MR and HRQoL in newly diagnosed patients with $\mathrm{CP} C \mathrm{CML}$ receiving TKI treatment in the BFORE trial, using a pooled analysis of the bosutinib and imatinib arms. To our knowledge, this is the first detailed investigation of the association between efficacy outcomes and HRQoL in this patient population using data from a randomized phase 3 clinical trial.

\section{Methods}

\section{BFORE study design}

As previously described [2], patients eligible for the BFORE study were aged $\geq 18$ years and had previously untreated $\mathrm{CP}$ CML, with a molecular diagnosis within the previous 6 months, and an Eastern Cooperative Oncology Group performance status of 0 or 1 . Patients were randomized 1:1 to receive either bosutinib $400 \mathrm{mg}$ once daily or imatinib $400 \mathrm{mg}$ once daily. Treatment is continued for 5 years until the end of the study or until treatment failure, unacceptable toxicity, death, or withdrawal of consent. The study was conducted in accordance with the Declaration of Helsinki, the protocol was approved by the Institutional Review Board at each study center, and all patients provided written informed consent. The trial is registered on ClinicalTrials.gov (NCT02130557).

\section{MR and HRQOL assessments}

MR was centrally assessed (MolecularMD, Portland, OR) by reverse transcription polymerase chain reaction (RT-PCR) using peripheral blood collected at baseline, every 3 months for the first 24 months of treatment, and every 6 months thereafter, and was evaluated on the international scale $[2,16]$.

HRQoL was assessed using the patient-reported FACT-Leu (version 4) questionnaire comprising 4 general HRQoL domains (physical, social, emotional, and functional well-being) and a leukemia-specific domain (Table 1) [2, 8, 9, 17, 18]. Each item in the questionnaire was scored from 0 to 4 , with higher scores indicating better HRQoL. Three second-order aggregated domains were based on the first-order general HRQoL and leukemia-specific domains: the FACT-General (FACT-G) total score is the sum of the physical, social, emotional, and functional well-being domain scores; the FACTLeu total score is the sum of the FACT-G total and leukemiaspecific domain scores; and the trial outcome index (TOI)FACT-Leu score is the sum of the physical well-being, functional well-being, and leukemia-specific domain scores, and represents a convenient summary index for clinical trials by assessing outcomes that are likely to change rapidly in response to treatment [18] (Table 1). The minimal important difference (MID), a change that is clinically meaningful to a patient, has been defined as $2-3$ points for physical well-being, 2 points for emotional well-being, 2-3 points for functional well-being, 4-7 points for leukemia-specific, 3-7 points for FACT-G, 6-12 points for the FACT-Leu total, and 5-6 points for TOI-FACT-Leu scores [8]; the MID for social well-being has not been defined. Patients were asked to complete FACT-Leu questionnaires at baseline, every 3 months for the first 24 months of treatment, every 6 months thereafter, and at treatment completion [11].

\section{Statistical analysis}

Data from the ITT population of patients with newly diagnosed CP CML in both arms of the BFORE trial (bosutinib and imatinib; $n=536$ ) were used to examine relationships between MR and patient-reported HRQoL (as measured by FACT-Leu). MR values were represented by a log-reduction scale [19] as a continuous variable.

A repeated-measures longitudinal model $[20,21]$ was used to estimate the overall relationships between MR logreduction (MRLR) score (from screening to 24 months) as a predictor and each FACT-Leu domain score as an outcome. 
Table $1 \quad$ FACT-Leu questionnaire [18]

\begin{tabular}{|c|c|c|c|}
\hline Domain & $\begin{array}{l}\text { Items, } \\
n\end{array}$ & $\begin{array}{l}\text { Score, } \\
\text { range }\end{array}$ & Questions \\
\hline Physical well-being & 7 & $0-28$ & $\begin{array}{l}\text { - I have a lack of energy } \\
\text { - I have nausea } \\
\text { - Because of my physical condition, I have trouble meeting the needs of my family } \\
\text { - I have pain } \\
\text { - I am bothered by side effects of treatment } \\
\text { - I feel ill } \\
\text { - I am forced to spend time in bed }\end{array}$ \\
\hline Social well-being & 7 & $0-28$ & $\begin{array}{l}\text { - I feel close to my friends } \\
\text { - I get emotional support from my family } \\
\text { - I get support from my friends } \\
\text { - My family has accepted my illness } \\
\text { - I am satisfied with family communication about my illness } \\
\text { - I feel close to my partner (or the person who is my main support) } \\
\text { - I am satisfied with my sex life }\end{array}$ \\
\hline $\begin{array}{l}\text { Emotional } \\
\text { well-being }\end{array}$ & 6 & $0-24$ & $\begin{array}{l}\text { - I feel sad } \\
\text { - I am satisfied with how I am coping with my illness } \\
\text { - I am losing hope in the fight against my illness } \\
\text { - I feel nervous } \\
\text { - I worry about dying } \\
\text { - I worry that my condition will get worse }\end{array}$ \\
\hline $\begin{array}{l}\text { Functional } \\
\text { well-being }\end{array}$ & 7 & $0-28$ & $\begin{array}{l}\text { - I am able to work (include work at home) } \\
\text { - My work (include work at home) is fulfilling } \\
\text { - I am able to enjoy life } \\
\text { - I have accepted my illness } \\
\text { - I am sleeping well } \\
\text { - I am enjoying the things I usually do for fun } \\
\text { - I am content with the quality of my life right now }\end{array}$ \\
\hline Leukemia-specific & 17 & $0-68$ & $\begin{array}{l}\text { - I am bothered by fevers } \\
\text { - I have certain parts of my body where I experience significant pain } \\
\text { - I am bothered by the chills } \\
\text { - I have night sweats } \\
\text { - I am bothered by lumps or swelling in certain parts of my body (e.g., neck, armpits, or groin) } \\
\text { - I bleed easily } \\
\text { - I bruise easily } \\
\text { - I feel weak all over } \\
\text { - I get tired easily } \\
\text { - I am losing weight } \\
\text { - I have a good appetite } \\
\text { - I am able to do my usual activities } \\
\text { - I worry about getting infections } \\
\text { - I feel uncertain about my future health } \\
\text { - I worry that I might get new symptoms of my illness } \\
\text { - I have emotional ups and downs } \\
\text { - I feel isolated from others because of my illness or treatment }\end{array}$ \\
\hline Aggregated domain & $\begin{array}{l}\text { Items, } \\
n\end{array}$ & $\begin{array}{l}\text { Score, } \\
\text { range }\end{array}$ & Calculation \\
\hline FACT-G total & 27 & $0-108$ & $\begin{array}{l}\text { Sum of the physical well-being, social well-being, emotional well-being, and functional well-being } \\
\text { scores }\end{array}$ \\
\hline FACT-Leu total & 44 & $0-176$ & Sum of the FACT-G total and the leukemia-specific scores \\
\hline TOI-FACT-Leu & 31 & $0-124$ & Sum of the physical well-being, functional well-being, and the leukemia-specific scores \\
\hline
\end{tabular}

FACT-G Functional Assessment of Cancer Therapy-General, FACT-Leu Functional Assessment of Cancer Therapy-Leukemia, TOI trial outcome index

This model incorporated all available data, generally using a heterogeneous autoregressive covariance structure to account for the correlated measurements (i.e., error terms) over time coming from the same individual, where the mechanism of missing data is at random [20,21]. Even if a patient had a missing observation at a particular post-baseline assessment, the completed post-baseline scores were still considered. To study the appropriateness of the linear approximation of the relationship between predictor and outcome, the model was also evaluated with MRLR score (from screening to 
24 months) as a categorical variable, in which MRLR values were rounded to the nearest 0.5 points.

An (standardized) effect size of 0.2 was considered small (i.e., the difference in means of 0.2 baseline standard deviation units), 0.5 was medium, and 0.8 was large; a value of $\sim 0.1$ was considered trivial $[22,23]$. Midpoints between values of $0.1,0.2,0.5$, and 0.8 were used to create categorization intervals for effect size. In a systematic review of PRO studies, an effect size of $\sim 0.5$ was determined to be the threshold for detecting changes in HRQoL [24].

\section{Results}

\section{Relationships between MR and HRQOL in the BFORE trial population}

Evaluation of the MRLR score as a continuous versus categorical variable indicated that the linearity assumption for the relationship between MRLR score and FACT-Leu total score was appropriate (Fig. 1). There was some visible departure from linearity for the MRLR value of -5 and to some extent for the value of -4.5 . This related to the relatively small number of available observations in the model with MRLR score as a categorical predictor, e.g., only $9(0.34 \%)$ observations with an MRLR score of -5 of 2668 total observations available and used in the analysis. Relationships between MRLR

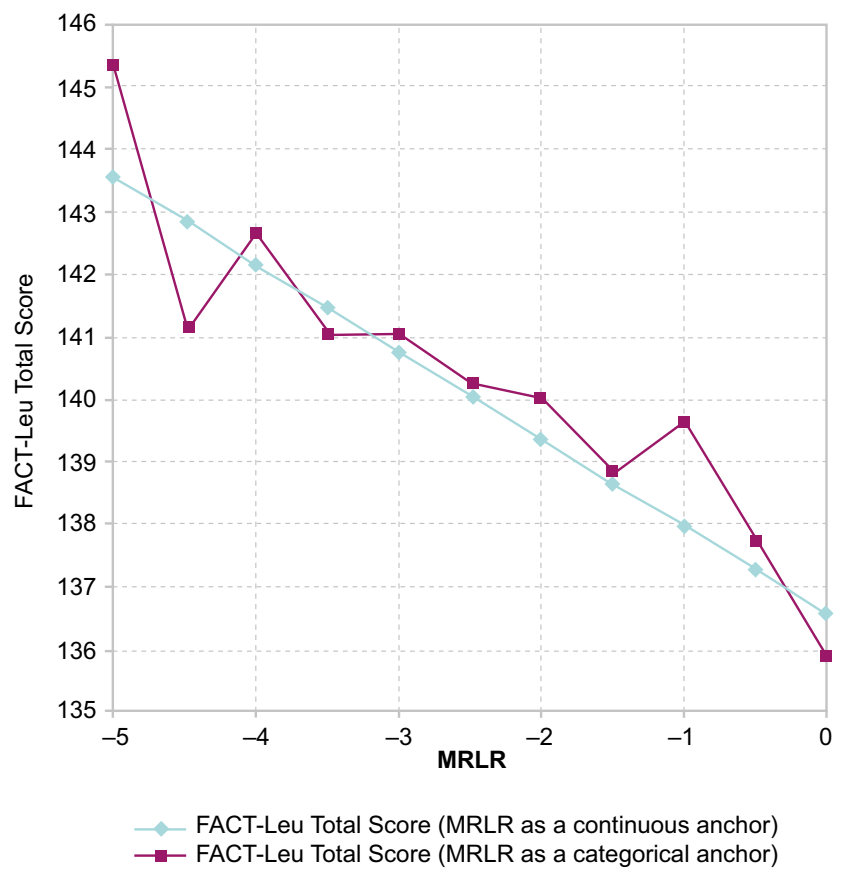

Fig. 1 Relationship between MRLR score and FACT-Leu total score in the BFORE trial population. FACT-Leu Functional Assessment of Cancer Therapy-Leukemia, MRLR molecular response log-reduction score and other FACT-Leu domain and aggregated domain scores exhibited similar patterns (data not shown).

\section{Differences in HRQOL according to MR level and interpretation of differences (effect size)}

The differences in estimated mean FACT-Leu domain and aggregated domain scores corresponding to MRLR values of $-5\left(\mathrm{MR}^{5}\right),-3(\mathrm{MMR})$, and $-1\left(\mathrm{MR}^{1}\right)$ versus MRLR value of 0 (standardized baseline; no response) in the context of their respective MIDs are shown in Fig. 2; social well-being, for which the MID has not been defined, is not shown. Based on the linear model, FACT-Leu total score differences corresponding to $\mathrm{MR}^{5}, \mathrm{MMR}$, and $\mathrm{MR}^{1}$ were significant $(p<0.0001)$. Differences corresponding to $\mathrm{MR}^{5}$, MMR, and $\mathrm{MR}^{1}$ were significant $(p<0.05)$ for FACT-G total, emotional well-being, functional well-being, leukemia-specific, and TOI-FACT-Leu scores, but were not significant for physical or social well-being scores. Only patients who achieved a deep MR $\left(\mathrm{MR}^{5}\right)$ exceeded the MID for the FACT-Leu total, FACT-G total, and TOI-FACT-Leu scores. The MID was not reached for physical well-being, emotional well-being, functional well-being, or leukemia-specific scores, regardless of depth of response.

Interpretation of differences, based on effect size, in FACTLeu domain and aggregated domain scores according to MR level is shown in Fig. 3. MR had the most robust relationships with emotional well-being and leukemia-specific scores, showing medium, small, and trivial differences associated with $\mathrm{MR}^{5}, \mathrm{MMR}$, and $\mathrm{MR}^{1}$, respectively. Differences in estimated mean FACT-Leu total and TOI-FACT-Leu scores were small for $\mathrm{MR}^{5}$ and MMR and trivial for $\mathrm{MR}^{1}$. The effect size of the difference in FACT-Leu total score corresponding to $\mathrm{MR}^{5}$ versus $\mathrm{MR}^{1}$ was 0.24 , which can be interpreted as small. For FACT-G total and functional well-being scores, differences associated with $\mathrm{MR}^{5}$ were small, and those associated with MMR and $\mathrm{MR}^{1}$ were trivial. MR had the weakest relationships with physical well-being and social well-being, where all differences were considered trivial.

\section{Discussion}

Treatment with TKIs that target Bcr-Abl1 has transformed CP CML to a chronic condition with normal life expectancy in most affected patients [1]. Given the need for lifelong therapy, HRQoL from the patient perspective has become a key component of clinical decision-making [25], in addition to response to individual therapies. Although evaluation of HRQoL through PRO assessments in clinical trials for CML is limited [7], studies of bosutinib have routinely collected PRO data to assess the symptom burden and functional health status of patients [8-11]. In the phase 3 BFORE trial of 


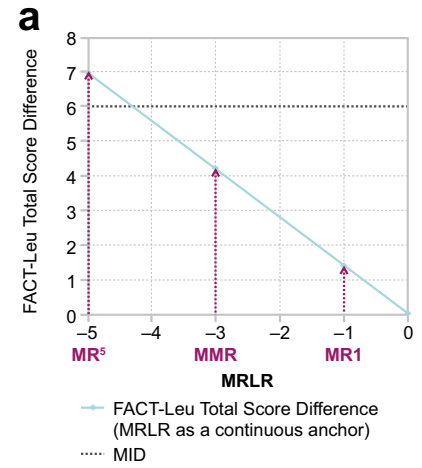

b

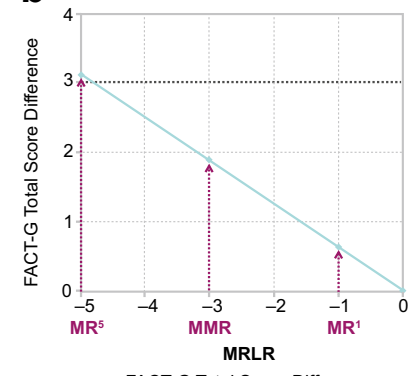

FACT-G Total Score Difference (MRLR as a continuous anchor)

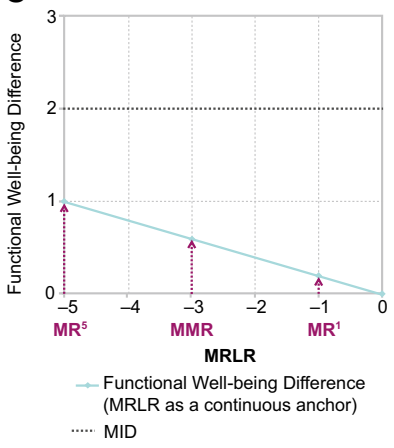$$
\text { ..... MID }
$$

Fig. 2 Differences in scores according to MR level for (a) FACT-Leu total, (b) FACT-G total, (c) physical well-being, (d) emotional well-being, (e) functional well-being, (f) leukemia-specific, and (g) TOI-FACT-Leu domains. FACT-G Functional Assessment of Cancer Therapy-General,

C

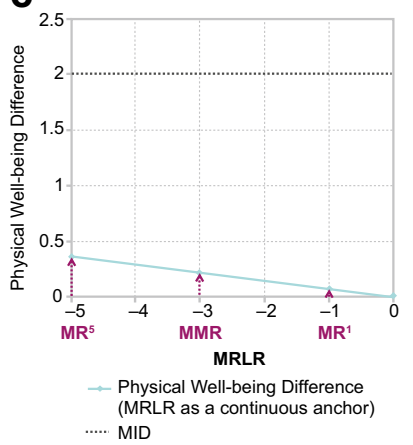

d

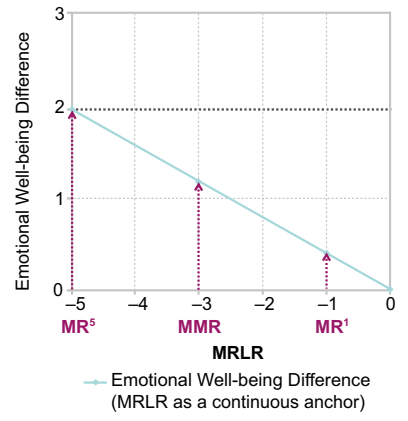

MID f

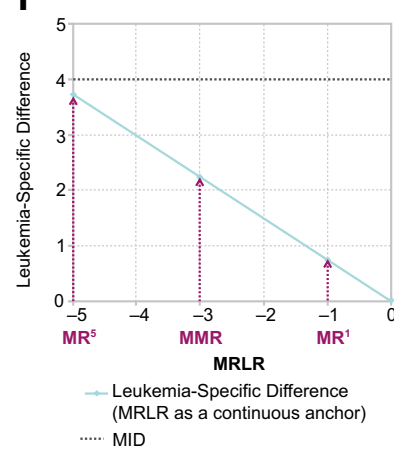

g

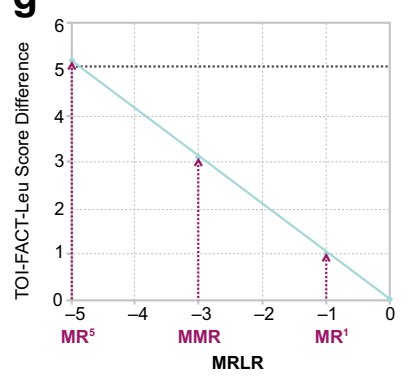

TOI-FACT-Leu Score Difference (MRLR as a continuous anchor) ...... MID

FACT-Leu Functional Assessment of Cancer Therapy-Leukemia, MID minimal important difference, $M M R$ major molecular response, $M R$ molecular response, MRLR molecular response log-reduction, TOI trial outcome index

Fig. 3 Comparison of the relationships between MR and FACT-Leu domain and aggregated domain scores according to MR level (effect size ${ }^{\mathrm{a}}$ ). FACT-G Functional Assessment of Cancer Therapy-General, FACT-Leu Functional Assessment of Cancer Therapy-Leukemia, $M M R$ major molecular response, $M R$ molecular response, TOI trial outcome index. ${ }^{\text {a }} \mathrm{A}$ (standardized) effect size of 0.2 is considered small (i.e., the difference in means being 0.2 baseline standard deviation units), 0.5 medium, and 0.8 large; a value of $\sim 0.1$ is trivial; midpoints between values of 0.1 , $0.2,0.5$, and 0.8 were used to create categorization intervals for effect size

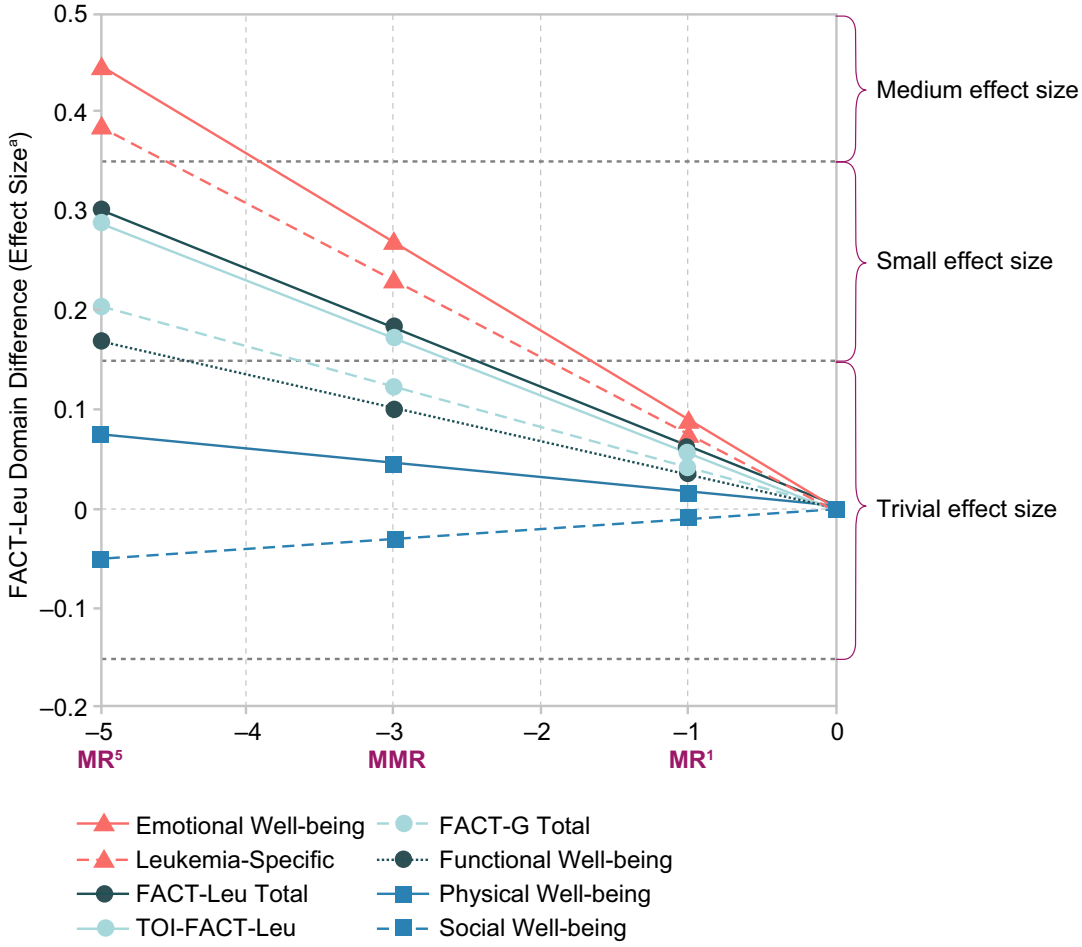


bosutinib versus imatinib in patients with newly diagnosed CP CML, similar degrees of improvement or preservation of HRQoL were seen in both treatment arms after 12 months [11]. The robust PRO dataset from the BFORE trial provided an exceptional opportunity to longitudinally analyze HRQoL data in patients with CML and explore the relationship with efficacy outcomes independent of the individual TKI administered.

In the present analysis, the impact of MR with first-line bosutinib or imatinib on different dimensions of HRQoL was variable. For patients who achieved deep MR, emotional well-being, and leukemia-specific scores showed the greatest improvement, although the respective MIDs were not reached. In the initial PRO analysis from the BFORE trial, emotional well-being and leukemia-specific scores improved significantly from baseline to the 12-month time point in both the bosutinib and imatinib arms [11]. Significant improvements in emotional well-being and leukemia-specific scores were also seen in imatinib-resistant and imatinib-intolerant patients at various time points during treatment with second-line bosutinib, although correlations with efficacy in these populations have not been investigated $[8,10,26]$. Since the emotional well-being domain of the FACT-Leu questionnaire reflects patients' optimism about their condition, a positive effect on this score with a response to treatment seems logical.

Physical and social well-being had the weakest relationships with MR in our analysis. As nearly $40 \%$ of patients with CP CML are asymptomatic at diagnosis in developed countries [27], the minimal impact of response on physical wellbeing was not unexpected. In addition, the physical well-being domain of FACT-Leu incorporates the impact of treatment side effects [18]. As is common for patients with CP CML receiving TKIs, most patients in the BFORE trial experienced treatment-emergent adverse events [2], which may partially explain a lack of improvement in physical well-being during treatment. Results from a prior meta-analysis of studies that included FACT-G data for patients with cancer suggested that social well-being, which measures support from family and friends and relationship fulfillment, may be less affected by disease or treatment outcomes than other FACT-G domains [28], as was seen here. The positive effect of a deep MR on emotional well-being and leukemia-specific scores would be anticipated to outweigh the influence of TKI side effects on overall HRQoL over the long term; this could be an area of future investigation.

The trivial or small effect size associated with MR for most FACT-Leu domains may reflect the already high scores ("ceiling effect") for patients with CP CML at diagnosis, making HRQoL improvements during treatment difficult to detect even if a response is achieved. Accordingly, the prior analysis of HRQoL in the BFORE trial found no association between achievement of MMR at 12 months and FACT-Leu total score in the modified ITT population [11]. It is important to note that the FACT-Leu questionnaire was not designed to capture HRQoL features that are specific to patients with CML receiving TKI therapy and was not validated in this population [17]; thus, some items may not reflect the real-life experience of this patient group, resulting in decreased sensitivity to identify changes associated with treatment or response. Nevertheless, the current analysis indicates that even if small, there is evidence of a positive effect of response on HRQoL, with better levels of response corresponding to more noticeable HRQoL improvements. Moreover, we found here that patients with a deep MR experienced clinically meaningful changes in FACT-Leu total, FACT-G total, and TOI-FACT-Leu scores, based on the MID for each score.

Although patients with CP CML are expected to have relatively high HRQoL scores prior to treatment, especially those who are asymptomatic at diagnosis, studies have found that patients receiving long-term TKI treatment for CP CML experience inferior HRQoL than individuals in the general population [29, 30]. In one report [29], impaired HRQoL was reported in younger (aged $<60$ years) and female patients compared with a matched control group; however, in patients aged $\geq 60$ years who received long-term imatinib for $\mathrm{CP}$ CML, HRQoL was comparable to the general population. A later study [30] that found significantly compromised HRQoL in patients with CP CML who received imatinib, dasatinib, or nilotinib versus age- and gender-matched controls may have been limited by a smaller number of participants and a crosssectional study design. Despite these findings, prospective PRO assessment in patients with CP CML treated with TKIs has indicated that HRQoL is maintained or improved from baseline during therapy [8-11, 31-34].

Response, including deep MR, to TKI treatment in patients with CP CML can be influenced by numerous factors, such as risk score, sex, adherence, and dose intensity [35]. Likewise, HRQoL can be affected by patient and treatment characteristics, including age, sex, comorbidities, and the side effects of the TKIs used to treat CML [29, 36-40]. It is also expected that these factors may influence the relationship between MR and FACT-Leu total score on an individual patient level. Although beyond the scope of the present analysis, this is a topic of interest for further research.

To our knowledge, the present analysis is the first rigorous exploration of relationships between efficacy outcomes and on-treatment HRQoL in patients with newly diagnosed CP CML from a large, prospective, multinational clinical trial. In a single-center study of 59 patients who received first-line nilotinib or imatinib in the phase 3 ENESTchina trial, optimal responses at 6 and 12 months per European LeukemiaNet guidelines [19] were associated with improvements in several HRQoL outcomes, measured with the Short Form 36 Health Survey (SF-36) questionnaire [41]. A longitudinal HRQoL analysis conducted as part of the GIMEMA trial of nilotinib for treatment of newly diagnosed CP CML reported 
improvements over time for physical functioning, role functioning, and fatigue, as assessed by the European Organization for Research and Treatment of Cancer Quality of Life Questionnaire-Core 30 (EORTC QLQ-C30); patients who reported greater physical fatigue prior to treatment were less likely to achieve an MMR, but association between response and on-treatment HRQoL was not assessed [33].

Analyses of HRQoL in clinical trials should be considered in the context of potential methodological issues. In the BFORE trial, PRO data were not collected from patients who discontinued from the trial, which is a common limitation of HRQoL analyses from clinical studies [25]. As of the data cutoff date for the primary analysis of the BFORE trial, 5 $(1.9 \%)$ patients in the bosutinib arm and $16(6.0 \%)$ in the imatinib arm had discontinued treatment due to suboptimal response or treatment failure [2]; thus, HRQoL could no longer be assessed longitudinally for these non-responders. In addition, there were relatively few observations in the model with an MRLR score of -5 . As previously reported, FACTLeu questionnaire completion rates in the BFORE trial were > $80 \%$ up to month 9 in the bosutinib arm and up to month 6 in the imatinib arm [11], and thus PRO compliance was not a methodological concern here [25]. Regarding sample size considerations [25], all available data from the BFORE trial, i.e., both treatment arms, were pooled to provide a large dataset for investigation of the general relationship between MR and HRQoL.

In conclusion, we found variable impact of clinical improvement on different dimensions of HRQoL in patients with newly diagnosed CP CML in the phase 3 BFORE trial. Our results suggest that better response to TKI treatment is generally associated with improved HRQoL. For patients who achieved deep MR, emotional well-being and leukemiaspecific domains showed the greatest improvement, whereas social well-being and physical well-being domains had the weakest relationships with MR; these patterns hold for patients who achieve MMR, although with diminished effect sizes. Prospective assessment of HRQoL and linking these data to clinical outcomes, including efficacy endpoints, should be systematically explored in future trials of TKIs in patients with CML.

Acknowledgments Medical writing support was provided by Joanna Bloom, $\mathrm{PhD}$, of Engage Scientific Solutions and was funded by Pfizer.

Data sharing statement Upon request, and subject to certain criteria, conditions and exceptions (see https://www.pfizer.com/science/clinicaltrials/trial-data-and-results for more information), Pfizer will provide access to individual de-identified participant data from Pfizer-sponsored global interventional clinical studies conducted for medicines, vaccines, and medical devices (1) for indications that have been approved in the US and/or EU or (2) in programs that have been terminated (i.e., development for all indications has been discontinued). Pfizer will also consider requests for the protocol, data dictionary, and statistical analysis plan. Data may be requested from Pfizer trials 24 months after study completion.
The de-identified participant data will be made available to researchers whose proposals meet the research criteria and other conditions, and for which an exception does not apply, via a secure portal. To gain access, data requestors must enter into a data access agreement with Pfizer.

\section{Author contributions}

- Designed research: THB, AGB, JCC, and CM

- Collected data: THB, CG-P, AGB, JCC, and SI

- Analyzed and interpreted data: THB, CG-P, AGB, JCC, AV, AR, SI, and CM

- Performed statistical analysis: AGB and JCC

- Wrote the manuscript: all authors

Funding information Open Access funding provided by Projekt DEAL. This study was funded by Pfizer.

Compliance with ethical standards The study was conducted in accordance with the Declaration of Helsinki, the protocol was approved by the Institutional Review Board at each study center, and all patients provided written informed consent.

Conflict of interest Tim H. Brümmendorf reports consultancy within the past 2 years for Novartis, Pfizer, Ariad, Janssen, and Merck, research funding from Novartis and Pfizer, honoraria from Pfizer, and other relationships with Novartis. Carlo Gambacorti-Passerini reports consultancy within the past 2 years for Bristol-Myers Squibb, and research funding and honoraria from Pfizer. Andrew G. Bushmakin, Joseph C. Cappelleri, Andrea Viqueira, Arlene Reisman, and Carla Mamolo are employed by Pfizer and own stock in Pfizer. Susanne Isfort reports consultancy within the past 2 years for Novartis and Pfizer, and honoraria from Novartis, Pfizer, Bristol-Myers Squibb, and Ariad/Incyte.

Open Access This article is licensed under a Creative Commons Attribution 4.0 International License, which permits use, sharing, adaptation, distribution and reproduction in any medium or format, as long as you give appropriate credit to the original author(s) and the source, provide a link to the Creative Commons licence, and indicate if changes were made. The images or other third party material in this article are included in the article's Creative Commons licence, unless indicated otherwise in a credit line to the material. If material is not included in the article's Creative Commons licence and your intended use is not permitted by statutory regulation or exceeds the permitted use, you will need to obtain permission directly from the copyright holder. To view a copy of this licence, visit http://creativecommons.org/licenses/by/4.0/.

\section{References}

1. Bower H, Bjorkholm M, Dickman PW, Hoglund M, Lambert PC, Andersson TM (2016) Life expectancy of patients with chronic myeloid leukemia approaches the life expectancy of the general population. J Clin Oncol 34(24):2851-2857. https://doi.org/10. 1200/JCO.2015.66.2866

2. Cortes JE, Gambacorti-Passerini C, Deininger MW, Mauro MJ, Chuah C, Kim DW, Dyagil I, Glushko N, Milojkovic D, le Coutre P, Garcia-Gutierrez V, Reilly L, Jeynes-Ellis A, Leip E, Bardy-Bouxin N, Hochhaus A, Brummendorf TH (2018) Bosutinib versus imatinib for newly diagnosed chronic myeloid leukemia: results from the randomized BFORE trial. J Clin Oncol 36(3):231-237. https://doi.org/10.1200/JCO.2017.74.7162

3. Kantarjian H, Shah NP, Hochhaus A, Cortes J, Shah S, Ayala M, Moiraghi B, Shen Z, Mayer J, Pasquini R, Nakamae H, Huguet F, Boque C, Chuah C, Bleickardt E, Bradley-Garelik MB, Zhu C, 
Szatrowski T, Shapiro D, Baccarani M (2010) Dasatinib versus imatinib in newly diagnosed chronic-phase chronic myeloid leukemia. N Engl J Med 362(24):2260-2270. https://doi.org/10.1056/ NEJMoa1002315

4. Saglio G, Kim DW, Issaragrisil S, le Coutre P, Etienne G, Lobo C, Pasquini R, Clark RE, Hochhaus A, Hughes TP, Gallagher N, Hoenekopp A, Dong M, Haque A, Larson RA, Kantarjian HM, ENESTnd Investigators (2010) Nilotinib versus imatinib for newly diagnosed chronic myeloid leukemia. N Engl J Med 362(24):22512259. https://doi.org/10.1056/NEJMoa0912614

5. Mahon FX, Rea D, Guilhot J, Guilhot F, Huguet F, Nicolini F, Legros L, Charbonnier A, Guerci A, Varet B, Etienne G, Reiffers J, Rousselot P, Intergroupe Francais des Leucemies Myeloides C (2010) Discontinuation of imatinib in patients with chronic myeloid leukaemia who have maintained complete molecular remission for at least 2 years: the prospective, multicentre Stop Imatinib (STIM) trial. Lancet Oncol 11(11):1029-1035. https://doi.org/10.1016/ S1470-2045(10)70233-3

6. Saussele S, Richter J, Guilhot J, Gruber FX, Hjorth-Hansen H, Almeida A, Janssen J, Mayer J, Koskenvesa P, Panayiotidis P, Olsson-Stromberg U, Martinez-Lopez J, Rousselot P, Vestergaard $\mathrm{H}$, Ehrencrona H, Kairisto V, Machova Polakova K, Muller MC, Mustjoki S, Berger MG, Fabarius A, Hofmann WK, Hochhaus A, Pfirrmann M, Mahon FX, EURO-SKI investigators (2018) Discontinuation of tyrosine kinase inhibitor therapy in chronic myeloid leukaemia (EURO-SKI): a prespecified interim analysis of a prospective, multicentre, non-randomised, trial. Lancet Oncol 19(6):747-757. https://doi.org/10.1016/S1470-2045(18)30192-X

7. De Marchi F, Medeot M, Fanin R, Tiribelli M (2017) How could patient reported outcomes improve patient management in chronic myeloid leukemia? Expert Rev Hematol 10(1):9-14. https://doi. org/10.1080/17474086.2017.1262758

8. Trask PC, Cella D, Besson N, Kelly V, Masszi T, Kim DW (2012) Health-related quality of life of bosutinib (SKI-606) in imatinibresistant or imatinib-intolerant chronic phase chronic myeloid leukemia. Leuk Res 36(4):438-442. https://doi.org/10.1016/j.leukres. 2011.10.011

9. Whiteley J, Reisman A, Shapiro M, Cortes J, Cella D (2016) Health-related quality of life during bosutinib (SKI-606) therapy in patients with advanced chronic myeloid leukemia after imatinib failure. Curr Med Res Opin 32(8):1325-1334. https://doi.org/10. 1185/03007995.2016.1174108

10. Kantarjian HM, Mamolo CM, Gambacorti-Passerini C, Cortes JE, Brummendorf TH, Su Y, Reisman AL, Shapiro M, Lipton JH (2018) Long-term patient-reported outcomes from an open-label safety and efficacy study of bosutinib in Philadelphia chromosome-positive chronic myeloid leukemia patients resistant or intolerant to prior therapy. Cancer 124(3):587-595. https://doi. org/10.1002/cncr.31082

11. Cortes JE, Gambacorti-Passerini C, Deininger MW, Mauro MJ, Chuah C, Kim DW, Milojkovic D, le Coutre P, Garcia-Gutierrez V, Crescenzo R, Mamolo C, Reisman A, Hochhaus A, Brummendorf TH, BFORE Study Investigators (2019) Patientreported outcomes in the phase $3 \mathrm{BFORE}$ trial of bosutinib versus imatinib for newly diagnosed chronic phase chronic myeloid leukemia. J Cancer Res Clin Oncol 145(6):1589-1599. https://doi.org/ 10.1007/s00432-019-02894-3

12. Brümmendorf TH, Mamolo CM, Reisman A, Bushmakin AG, Cappelleri JC, Crescenzo RJ, DeAnnuntis L, Viquiera A, Cortes JE (2018) Impact of diarrhea on health-related quality of life: analysis of the phase 3 BFORE trial of bosutinib vs imatinib for newly diagnosed chronic phase chronic myeloid leukemia. Blood 132(Suppl 1):4264. https://doi.org/10.1182/blood-2018-99110152

13. Cortes JE, Mauro MJ, Deininger MWN, Chuah C, Kim D-W, Kota V, Lipton JH, Rousselot PH, Milojkovic D, Coutre PDL, Gutierrez
VG, Crescenzo RJ, Leip E, An F, Bouxin N, Hochhaus A, Brümmendorf TH, Gambacorti-Passerini C (2018) Bosutinib vs imatinib for newly diagnosed chronic myeloid leukemia in the BFORE trial: 24-month follow-up. J Clin Oncol 36(15_suppl): 7002. https://doi.org/10.1200/JCO.2018.36.15_suppl.7002

14. Khoury HJ, Gambacorti-Passerini C, Brummendorf TH (2018) Practical management of toxicities associated with bosutinib in patients with Philadelphia chromosome-positive chronic myeloid leukemia. Ann Oncol 29(3):578-587. https://doi.org/10.1093/annonc/ mdy019

15. Steegmann JL, Baccarani M, Breccia M, Casado LF, GarciaGutierrez V, Hochhaus A, Kim DW, Kim TD, Khoury HJ, Le Coutre P, Mayer J, Milojkovic D, Porkka K, Rea D, Rosti G, Saussele S, Hehlmann R, Clark RE (2016) European LeukemiaNet recommendations for the management and avoidance of adverse events of treatment in chronic myeloid leukaemia. Leukemia 30(8):1648-1671. https://doi.org/10.1038/leu.2016.104

16. Hughes T, Deininger M, Hochhaus A, Branford S, Radich J, Kaeda J, Baccarani M, Cortes J, Cross NC, Druker BJ, Gabert J, Grimwade D, Hehlmann R, Kamel-Reid S, Lipton JH, Longtine J, Martinelli G, Saglio G, Soverini S, Stock W, Goldman JM (2006) Monitoring CML patients responding to treatment with tyrosine kinase inhibitors: review and recommendations for harmonizing current methodology for detecting BCR-ABL transcripts and kinase domain mutations and for expressing results. Blood 108(1): 28-37. https://doi.org/10.1182/blood-2006-01-0092

17. Cella D, Jensen SE, Webster K, Hongyan D, Lai JS, Rosen S, Tallman MS, Yount S (2012) Measuring health-related quality of life in leukemia: the Functional Assessment of Cancer TherapyLeukemia (FACT-Leu) questionnaire. Value Health 15(8):10511058. https://doi.org/10.1016/j.jval.2012.08.2210

18. FACIT.org FACT-Leu (Version 4). https://www.facit.org/ FACITOrg/Questionnaires. Accessed 23 Aug 2019

19. Baccarani M, Deininger MW, Rosti G, Hochhaus A, Soverini S, Apperley JF, Cervantes F, Clark RE, Cortes JE, Guilhot F, HjorthHansen H, Hughes TP, Kantarjian HM, Kim DW, Larson RA, Lipton JH, Mahon FX, Martinelli G, Mayer J, Muller MC, Niederwieser D, Pane F, Radich JP, Rousselot P, Saglio G, Saussele S, Schiffer C, Silver R, Simonsson B, Steegmann JL, Goldman JM, Hehlmann R (2013) European LeukemiaNet recommendations for the management of chronic myeloid leukemia: 2013. Blood 122(6):872-884. https://doi.org/10.1182/blood-201305-501569

20. Fairclough DL (2010) Design and analysis of quality of life studies in clinical trials, 2nd edn. Chapman \& Hall/CRC, Boca Raton

21. Fitzmaurice GM, Laird NM, Ware JH (2011) Applied longitudinal analysis, 2nd edn. Wiley, Hoboken

22. Cohen J (1988) Statistical power analysis for the behavioral sciences. Lawrence Erlbaum Associates, Hillsdale

23. Cappelleri JC, Zou KH, Bushmakin AG, Alvir JMJ, Alemayehu D, Symonds T (2013) Patient-reported outcomes: measurement, implementation and interpretation. CRC Press, Boca Raton

24. Norman GR, Sloan JA, Wyrwich KW (2003) Interpretation of changes in health-related quality of life: the remarkable universality of half a standard deviation. Med Care 41(5):582-592. https://doi. org/10.1097/01.MLR.0000062554.74615.4C

25. Efficace F, Cannella L (2016) The value of quality of life assessment in chronic myeloid leukemia patients receiving tyrosine kinase inhibitors. Hematol Am Soc Hematol Educ Program 2016(1): 170-179. https://doi.org/10.1182/asheducation-2016.1.170

26. Gambacorti-Passerini C, Cortes JE, Lipton JH, Kantarjian HM, Kim DW, Schafhausen P, Crescenzo R, Bardy-Bouxin N, Shapiro M, Noonan K, Leip E, DeAnnuntis L, Brummendorf TH, Khoury HJ (2018) Safety and efficacy of second-line bosutinib for chronic phase chronic myeloid leukemia over a five-year period: final 
results of a phase I/II study. Haematologica 103(8):1298-1307. https://doi.org/10.3324/haematol.2017.171249

27. Cortes J (2004) Natural history and staging of chronic myelogenous leukemia. Hematol Oncol Clin North Am 18(3):569-584, viii. https://doi.org/10.1016/j.hoc.2004.03.011

28. King MT, Stockler MR, Cella DF, Osoba D, Eton DT, Thompson J, Eisenstein AR (2010) Meta-analysis provides evidence-based effect sizes for a cancer-specific quality-of-life questionnaire, the FACTG. J Clin Epidemiol 63(3):270-281. https://doi.org/10.1016/j. jclinepi.2009.05.001

29. Efficace F, Baccarani M, Breccia M, Alimena G, Rosti G, Cottone F, Deliliers GL, Barate C, Rossi AR, Fioritoni G, Luciano L, Turri D, Martino B, Di Raimondo F, Dabusti M, Bergamaschi M, Leoni P, Simula MP, Levato L, Ulisciani S, Veneri D, Sica S, Rambaldi A, Vignetti M, Mandelli F, Gimema (2011) Health-related quality of life in chronic myeloid leukemia patients receiving long-term therapy with imatinib compared with the general population. Blood 118(17):4554-4560. https://doi.org/10.1182/blood-2011-04347575

30. Phillips KM, Pinilla-Ibarz J, Sotomayor E, Lee MR, Jim HS, Small BJ, Sokol L, Lancet J, Tinsley S, Sweet K, Komrokji R, Jacobsen PB (2013) Quality of life outcomes in patients with chronic myeloid leukemia treated with tyrosine kinase inhibitors: a controlled comparison. Support Care Cancer 21(4):1097-1103. https://doi.org/10. 1007/s00520-012-1630-5

31. Aziz Z, Iqbal J, Aaqib M, Akram M, Saeed A (2011) Assessment of quality of life with imatinib mesylate as first-line treatment in chronic phase-chronic myeloid leukemia. Leuk Lymphoma 52(6): 1017-1023. https://doi.org/10.3109/10428194.2011.560310

32. Hahn EA, Glendenning GA, Sorensen MV, Hudgens SA, Druker BJ, Guilhot F, Larson RA, O’Brien SG, Dobrez DG, Hensley ML, Cella D, IRIS Investigators (2003) Quality of life in patients with newly diagnosed chronic phase chronic myeloid leukemia on imatinib versus interferon alfa plus low-dose cytarabine: results from the IRIS study. J Clin Oncol 21(11):2138-2146. https://doi.org/10. 1200/JCO.2003.12.154

33. Efficace F, Castagnetti F, Martino B, Breccia M, D'Adda M, Angelucci E, Stagno F, Cottone F, Malato A, Trabacchi E, Capalbo SF, Gobbi M, Visani G, Salvucci M, Capodanno I, Tosi P, Tiribelli M, Scortechini AR, Levato L, Maino E, Binotto G, Gugliotta G, Vignetti M, Baccarani M, Rosti G (2018) Healthrelated quality of life in patients with chronic myeloid leukemia receiving first-line therapy with nilotinib. Cancer 124(10):22282237. https://doi.org/10.1002/cncr.31323

34. Sacha T, Gora-Tybor J, Wasak-Szulkowska E, Kyrcz-Krzemien S, Medras E, Becht R, Bober G, Kotowska A, Waclaw J, Hellmann A (2017) Quality of life and adherence to therapy in patients with chronic myeloid leukemia treated with nilotinib as a second-line therapy: a multicenter prospective observational study. Clin Lymphoma Myeloma Leuk 17(5):283-295. https://doi.org/10. 1016/j.clml.2017.01.001

35. Mahon FX, Etienne G (2014) Deep molecular response in chronic myeloid leukemia: the new goal of therapy? Clin Cancer Res 20(2): 310-322. https://doi.org/10.1158/1078-0432.CCR-13-1988

36. Efficace F, Baccarani M, Breccia M, Cottone F, Alimena G, Deliliers GL, Barate C, Specchia G, Di Lorenzo R, Luciano L, Turri D, Martino B, Stagno F, Dabusti M, Bergamaschi M, Leoni P, Simula MP, Levato L, Fava C, Veneri D, Sica S, Rambaldi A, Rosti G, Vignetti M, Mandelli F (2013) Chronic fatigue is the most important factor limiting health-related quality of life of chronic myeloid leukemia patients treated with imatinib. Leukemia 27(7): 1511-1519. https://doi.org/10.1038/leu.2013.51

37. Guerin A, Chen L, Ionescu-Ittu R, Marynchenko M, Nitulescu R, Hiscock R, Keir C, Wu EQ (2014) Impact of low-grade adverse events on health-related quality of life in adult patients receiving imatinib or nilotinib for newly diagnosed Philadelphia chromosome positive chronic myelogenous leukemia in chronic phase. Curr Med Res Opin 30(11):2317-2328. https://doi.org/10.1185/03007995. 2014.944973

38. Kekale M, Peltoniemi M, Airaksinen M (2015) Patient-reported adverse drug reactions and their influence on adherence and quality of life of chronic myeloid leukemia patients on per oral tyrosine kinase inhibitor treatment. Patient Prefer Adherence 9:1733-1740. https://doi.org/10.2147/PPA.S92125

39. Efficace F, Rosti G, Breccia M, Cottone F, Giesinger JM, Stagno F, Iurlo A, Russo Rossi A, Luciano L, Martino B, Galimberti S, Turri D, Bergamaschi M, Tiribelli M, Fava C, Angelucci E, Mandelli F, Baccarani M (2016) The impact of comorbidity on health-related quality of life in elderly patients with chronic myeloid leukemia. Ann Hematol 95(2):211-219. https://doi.org/10.1007/s00277-0152541-6

40. Jiang Q, Wang HB, Yu L, Gale RP (2017) Variables associated with patient-reported outcomes in persons with chronic myeloid leukemia receiving tyrosine kinase-inhibitor therapy. J Cancer Res Clin Oncol 143(6):1013-1022. https://doi.org/10.1007/s00432-0172353-2

41. Yu L, Wang H, Milijkovic D, Huang X, Jiang Q (2018) Achieving optimal response at 12 months is associated with a better healthrelated quality of life in patients with chronic myeloid leukemia: a prospective, longitudinal, single center study. BMC Cancer 18(1): 782. https://doi.org/10.1186/s12885-018-4699-5

Publisher's note Springer Nature remains neutral with regard to jurisdictional claims in published maps and institutional affiliations. 